P33 (continued)

influence the food insecurity of low-income, food-insecure individuals with chronic diseases. Improvements on program focus, intensity of nutrition education, and adjustments to content could improve the impact of the program.

Funding: Regional Food Bank of Oklahoma

\section{P34 Faith-Based Nutrition and Physical Activity Interventions: A Systematic Review of the Literature with Future Recommendations}

Kimberlee Kinney, BS, skim13@vt.edu, Virginia Tech, 327 Wallace Hall, Blacksburg, VA 24061; Elena Serrano, PhD, Virginia Tech; Kathy Hosig, PhD, MPH, RD;

Jay Williams, PhD; Vivica Kraak, PhD, RD

Objective: Faith-based organizations (FBOs) offer a unique setting opportunity for delivering sustainable health promotion interventions targeting diet and physical activity lifestyle changes in communities. FBOs are deeply rooted within their local communities and strong social ties, giving them an ability to reach large proportion of the population. Previous reviews in this field have primarily focused on obesity-related research as it relates to African American populations or chronic disease. The purpose of this systematic review is to summarize the characteristics, methods, and strategies used in faithbased nutrition and physical activity health promotion interventions targeting all populations, in order to identify strengths and gaps, and to inform best practices for future initiatives.

Study Design, Setting, Participants, Intervention: Data collection and reporting was guided by PRISMA statement guidelines. Electronic databases were used to identify articles through February 2017. Eligible studies took place in a faith setting in the U.S., written in English, and included a nutrition or physical activity component to them. Chronic disease management, or those lacking an intervention component were excluded.

Outcome Measures and Analysis: Primary outcomes reported include study design, location, target population characteristics, faith denomination and faith components, relevant theories and frameworks used, and types of policy, systems and environmental (PSE) changes integrated into the interventions.

Results: Most studies targeted African Americans, located in urban areas. Several studies utilized peer educators to deliver the intervention, and incorporated policy, systems and environmental changes at the church level.

Conclusions and Implications: Multi-level interventions are key when working with FBOs to deliver health programming. More research is needed in rural areas, with non-Christian faith sectors, and in populations other than African Americans.

Funding: None
P35 Families Get Fit with Family, Food, \& Fun!: Evaluation of a Four-Week Family Wellness Program

Blair Murphy, MPA, CPT, blair.murphy@bswhealth.org, Baylor Scott \& White Health McLane Children's, 1901 SW HK Dodgen Loop, MS-CK-300, 3.221, Temple, TX 76513; Dell Ingram-Walker, MBA, Baylor Scott \& White Health McLane Children's; Jason Culp; Lea Mallett, PhD

Objective: To improve family wellness, including dietary behaviors and physical fitness.

Study Design, Setting, Participants, Intervention: Prospective cohort study to evaluate Family, Food, \& Fun!, a four-week family wellness education program in Killeen, TX for children of families with low socioeconomic status, ages 8-15, and their families, from November 2015 to November 2016. Families receive health lessons reinforced with interactive cooking and fitness demonstrations.

Outcome Measures and Analysis: Participant data collected at weeks 1 and 4, and 12 months includes a fitness assessment, biometrics, physical activity tracker data, and daily food/activity journals. Outcomes include body mass index (BMI), healthy eating habits, and physical activity. Bivariate analyses was performed to compare outcomes from baseline to week 4 .

Results: Fourty-one families (62 children, 41 adults) have completed the program to date. Thirty-five percent of families were Caucasian and $44 \%$ were Black. Median age of the children was 10 years. Median weight at baseline was 101 lbs (range, 49-322) and average child BMI was $22( \pm 7.4)$. Median parent BMI at baseline was 33 $( \pm 9.5)$. Change in BMI was not significant. Children significantly improved on the timed half-mile $(\mathrm{p}=0.042)$ and number of sit-ups in one minute $(\mathrm{p}=0.012)$ at the 4 week fitness assessment. Parents reported an increase in days child was active for $60 \mathrm{mi}-$ nutes $(\mathrm{p}=0.022)$.

Conclusions and Implications: Family, Food, \& Fun! improved fitness among children in 4 weeks. Longitudinal data collection and analyses are ongoing to further investigate above and additional outcomes over a longer term. The results of this program will be applicable to healthcare and community organizations focused on wellness promotion and obesity prevention.

Funding: None

\section{P36 Family Education Program Improves Diet, Family Meals, and Physical Activity with Interactive Demonstrations}

Blair Murphy, MPA, CPT, blair.murphy@bswhealth.org, Baylor Scott \& White Health McLane Children's, 1901 SW HK Dodgen Loop, MS-CK-300, 3.221, Temple, TX 76513; Lea Mallett, PhD, Baylor Scott \& White Health McLane Children's; Michelle Law, PhD, RD

Objective: To improve dietary behaviors (reduce sugar and salt intake, read nutrition labels, increase 